\title{
Brand asset management in the age of Google
}

\section{Johanna Shalhoub}

is Vice President, Business Development Brand Amplifire ${ }^{\mathrm{TM}}$ for Siegel+Gale. Her job is to facilitate the incorporation of the breakthrough, easy-to-use, Brand Amplifire system into the day-to-day and even hour-to-hour needs of today's brand manager and team. If you can use Google, you can manage your brand with Brand Amplifire.

\section{Howard Belk}

is Co-President of Siegel+Gale, and shares operating responsibility for the overall performance of the firm with partner David Srere. Slightly more fun than counting and allotting the corporate coins, however, is his role as Chief Creative Officer and anointed High Priest of Unorthodoxy. It is Howard's job to challenge S+G's corporate branding, advertising, interactive, and simplification creative teams to live up to and give fresh expression to the credo of our founder Alan Siegel — Simple is Smart. At Siegel+Gale, simple means brand expressions that are clear, fresh, honest, useful, and inspiring.

\section{J.P. Terry \\ is the CEO of SmartDoc Technologies, a leading company in the field of dynamic document solutions. Prior to SmartDoc Technologies, J.P. founded one of the most successful first-generation brand asset management consultants, BrandWizard Technologies. Mr. Terry writes extensively on technology and design issues including his recent book, Creating Dynamic Forms with Adobe LiveCycle Designer on Adobe Press.}

Keywords: brand asset management, Google, DAM, metadata, brand management, asset management

Abstract What started as brand guidelines in the format of binders that would collect dust on bookshelves has transformed into an industry of its own. For almost 15 years now, brand asset management (BAM) solutions have been helping companies worldwide manage a company's most valuable asset-its brand. As technology improved and the web transformed the way business was done, BAM solutions turned to the web to enable easy access to a brand's database. However, many of these first-generation systems, were not successful because they were difficult to use and they quickly became outdated. Enter Google. In an age where anyone can go online and find images for just about anything with a mouse-click, there is a need to simplify this essential solution. The following paper takes a look at how BAM has transformed and how Google technology is being leveraged to provide easy-to-use tools that can be used by anyone in your organization to ensure your brand is being expressed the way it was intended.

Journal of Digital Asset Management (2007) 3, 269-273. doi:10.1057/palgrave.dam.3650094

Johanna Shalhoub Siegel+Gale, 437 Madison Ave, 12th floor, NY 10022, NewYork.

Tel: +12128176650 Email: jshalhoub@ siegelgale.com

\section{FIRST-GENERATION BAM}

First-generation brand asset management (BAM) systems are tightly structured web-content repositories, a natural progression from their origins as hierarchically structured guidelines books.

As Internet technology became available, brand managers explored ways to centralize their guidelines online so that they could be distributed efficiently across their organizations. Once that goal was realized, BAM systems were extended to include asset management and document creation tools.

\section{Structured content}

While first-generation BAM systems successfully fulfill their original purpose, their model of structured content inherently limits them. Systems that require structured content are limited for many reasons:

- They are not easily extensible. New content and features always need to be worked into the existing structure.

- They require a complicated implementation process. An entire hierarchical structure needs to be 
developed to navigate and organize the content. Usually, all the assets to be contained in the system must be available or at least planned for in advance.

- They are designed to distribute information topdown. The presumption is that regular end users have no valuable feedback. This also puts an additional barrier to adoption.

- They are limited to the knowledge and experience of a small group of managers. That group cannot possibly predict every scenario of brand usage that occurs in the real world.

- They are expensive to maintain. As the system grows in content, it grows in complexity. The increased complexity often results in additional costs that were not planned for and can diminish overall expected return on investment (ROI).

- They are centralized and quickly outdated. As the brand assets make their way through an organization, they are stored on local machines and quickly disconnected from the brand management system that was put into place to monitor them. Over time, this can result in utilizing outdated assets.

- The structure of the system and the user interface often need an explanation in order for new users to know where to look for the assets they need.

\section{NEXT-GENERATION BAM}

Next-generation BAM systems are built on what may be perceived as a counter-intuitive premise that less structure is better. Traditional thinking holds that structured and centralized content provides more control over brand materials, translating into a better protected brand. The reality is that structure creates the increased complexities discussed above.

The best way to protect a brand is to provide the right materials to the people who communicate the brand day-to-day. Ultimately, this is the group that will use the system on a regular basis and their utilization of the system is critical to its success.

That means providing an open and userfriendly system. If it is difficult for people to access the proper assets, they will create their own work-arounds, which can be detrimental. However, if the system makes it easy to stay "on-brand," people will not only use the system, but they will find ways to express the brand that could not have been anticipated.
Given the correctly aligned branding tools in a timely manner, your people can bring their broad range of talents to bear on exploiting the latest marketing opportunity, rather than merely surviving it.

\section{Branding at the speed of business}

People use a BAM system to browse and locate the brand assets that they require, typically for a specific project that needs to be finished immediately. An enterprise search engine is the perfect vehicle to deliver results for real-world BAM scenarios, if it works well.

To be useful, an enterprise search-based BAM system must excel in:

- Performance

- Relevancy

- Ease-of-use.

Performance is an often overlooked factor in the effectiveness of BAM systems. We frequently hear that a system with great features is unused because it is too slow. It is great to have sophisticated sorting and previewing features, but if the page does not load in under a second, users will lose patience and abandon the search. This will only increase if your users are accessing the system across continents when load times are slow.

In the age of Google, expectations are high. Searches must return instant, relevant results right at the top of the result set. This requires a great relevancy algorithm in the search technology, since ultimately providing relevant search results is a partnership between the technology and the people responsible for the system. A great relevancy algorithm is incomplete without a significant set of searchable content.

Ease-of-use is also critical to the system's success. This is accomplished by keeping the user interface clear and uncluttered. The system's design must uphold the main goal of retrieving files at the forefront.

The Siegel+Gale Brand Amplifire ${ }^{\mathrm{TM}}$ BAM system utilizes the Google Search Appliance to provide incredibly fast performance, the best relevancy algorithm in the world, and a simple search result interface. Additionally, with the Google Appliance, Amplifire has the benefit of a low cost of entry, simple implementation and 
configuration, and the cachet of the Google name. Amplifire, with the Google Appliance, is the first choice for systems that are branding at the speed of business.

\section{GOOGLE SEARCH}

At the core of the Amplifire BAM system, the Google Appliance provides powerful capabilities in three areas: indexing, searching, and serving. The Google Appliance crawls file content (indexing), accepts sophisticated search queries (searching) and returns relevant results (serving). However, in the context of BAM, there are more considerations.

First, there is the graphical nature of many BAM files such as logos. These cannot simply be thrown into a system and indexed since there is no text content to index.

Second, there is the issue of extensibility. The Google Appliance can serve links for file downloads, but many BAM systems feature addon tools for graphics and document processing. How can a user access these tools if the content is available only through search?
Third is the problem of segmentation and access privileges, such as power user, external user, administrator, etc. Many companies rely on outside agencies and print vendors to work with their marketing materials. These external parties must be able to access the system. In addition, many organizations have regional offices that need to share some marketing materials but not others. Brand content must be segmented to reflect the types of assets that people may need to search for exclusively.

The Amplifire solution addresses these issues by employing a tightly coupled three-tiered architecture model that combines document and image processing, database access controls, and metadata filters to extend or "amplify" the Google search.

\section{Application architecture}

The top level is the file processing tier. Before content becomes searchable, it is processed to extract metadata, generate preview imagery, and process documents for customization. This
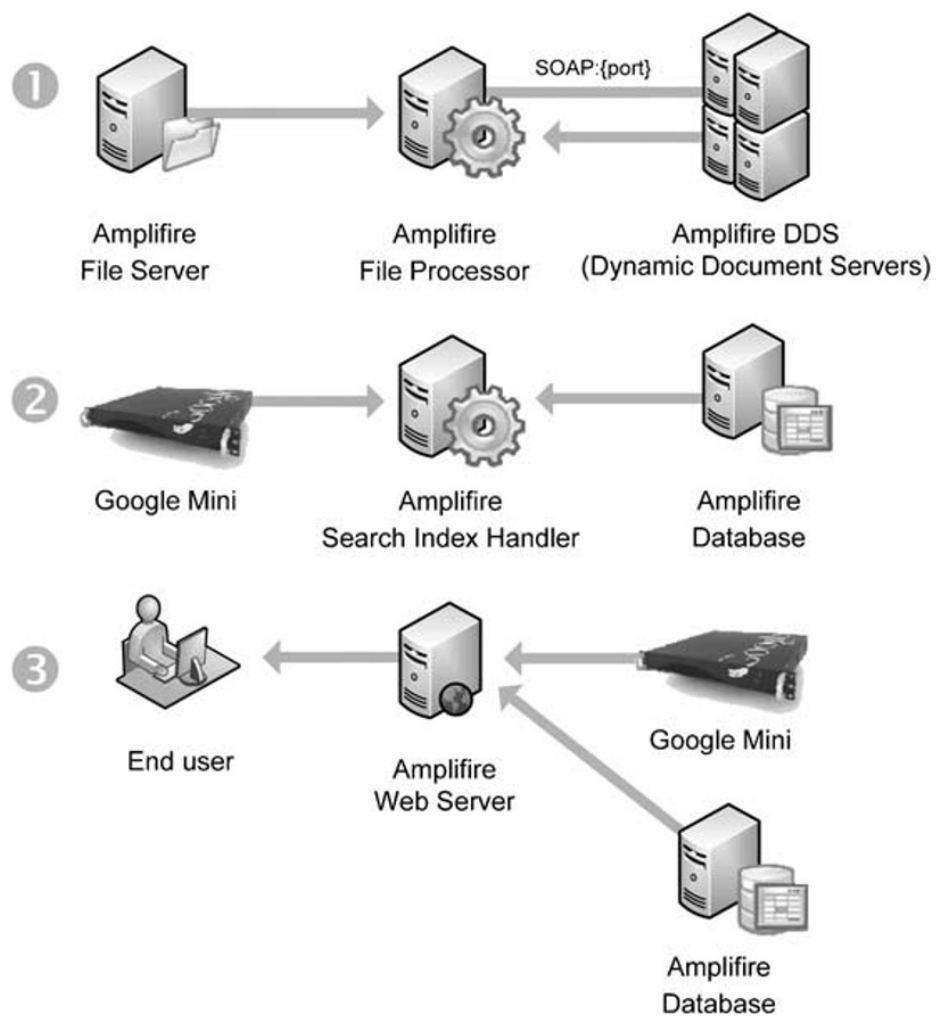

Figure 1: The Amplifire three-tiered architecture for indexing and serving files 


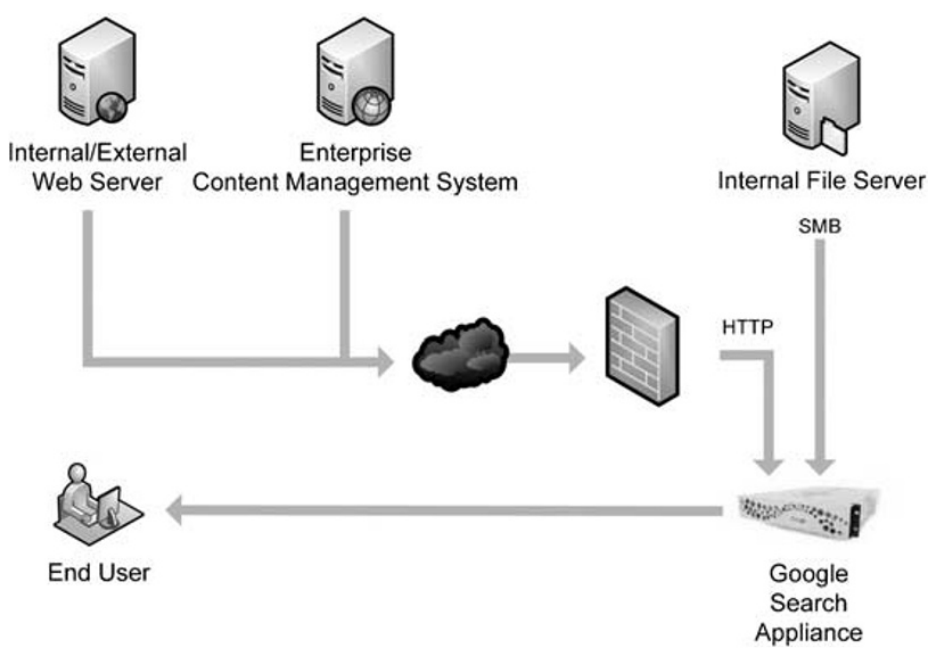

Figure 2: Searching enterprise content with the Google Search Appliance

involves using specialized Dynamic Document Servers (DDS), Adobe XMP-based server applications, and Microsoft Office server components.

The second level is the search index handler. This is a controlled environment for generating searchable representations of wiki content and metadata for the search crawler.

The third is the web application that serves the results. This web application retrieves results directly from the search appliance and then correlates the results to database information and add-on features (Figure 1).

\section{Enterprise architecture}

See Figure 2.

\section{WIKI CONTENT FOR BRAND MANAGEMENT - THE BRANDWIKI}

Next-generation BAM systems need to be more than top-down asset repositories. Letting go of the central control of assets opens the system to collaboration. When even Time magazine recognizes "You" as the person of the year, we must respect this shift and facilitate collaboration by those using the system and its assets most frequently to determine the system's success. This is solved by utilizing a wiki-style editing model where everyone can contribute to content and files.
The BrandWiki is a collaborative, wiki style, content creation and editing application that works with brand team supervision. The BrandWiki gives end users an outlet to share their experiences and find other sources of information (Figure 3).

A BrandWiki can help to:

- Distribute the information and asset-gathering burden across the entire organization.

- Give everyone in the organization a sense of ownership in the system.

- Allow subject matter experts to expand the topic to include a higher level of detail than would be possible from a centralized management group.

- Share knowledge for real-world brand implementation scenarios across the organization.

A true next-generation BAM system is inclusive. Above all, next-generation BAM is really about brand management widening the circle to incorporate the talents of people who can contribute to advancing the brand.

Pairing the BrandWiki concept with Amplifire's Google Appliance-based system creates a perfect loop of information where content is: updated $>$ indexed $>$ searched $>$ and updated. This, along with oversight from an administration team that can assess search logs, review activity, and batch update content provides the perfect tool for BAM in today's age of the ubiquitous user-generated content. 


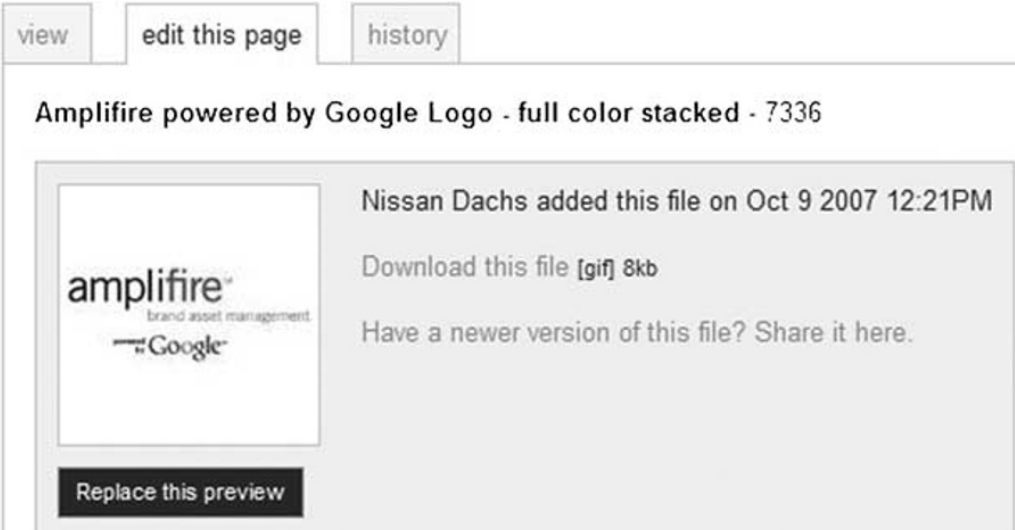

Show the properties of this file

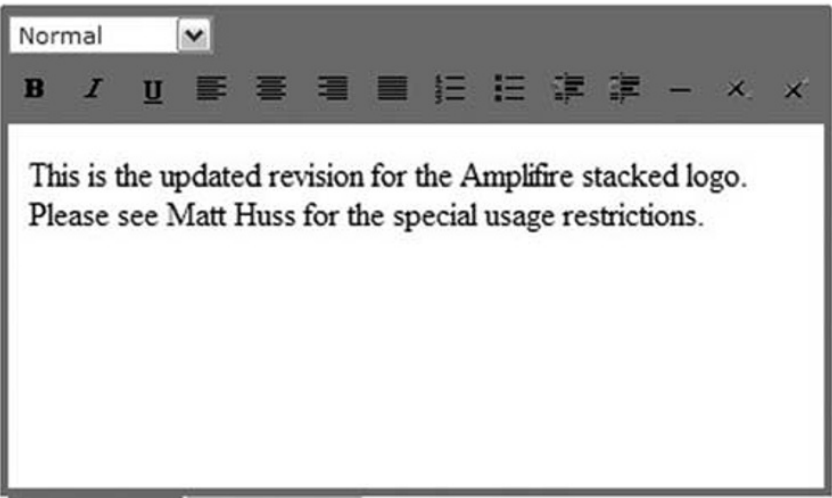

\section{$\triangle$ Design* 들 $H$ tml}

\section{Tags}

amplifire, eps, full color, logo, stacked

PUBLISH WIKI \& SAVE PROPERTES

Figure 3: The Siegel+Gale Amplifire system BrandWiki editing

\section{FOR FURTHER INFORMATION}

\section{Brand Amplifire ${ }^{\mathrm{TM}}$}

Brand Amplifire is a next-generation BAM tool based on the Google Search engine. Amplifire is a partnership between Siegel+Gale and SmartDoc Technologies. When you work on an Amplifire project, you will be working with the respective professionals from Siegel+Gale and SmartDoc Technologies. 